\title{
Key impact of finite-beta and fast ions in core and edge tokamak regions for the transition to advanced scenarios
}

\author{
J. Garcia ${ }^{1}$, C. Challis ${ }^{2}$, J. Citrin ${ }^{1,3}$, H. Doerk ${ }^{4}$, G. Giruzzi ${ }^{1}$, T. Görler ${ }^{4}$, F. Jenko ${ }^{4}$, P. Maget ${ }^{1}$ and \\ JET contributors*
}

\author{
JET-EFDA, Culham Science Centre, Abingdon, OX14 3DB, UK \\ ${ }^{1}$ CEA, IRFM, F-13108 Saint-Paul-lez-Durance, France. \\ ${ }^{3}$ FOM Institute DIFFER - Dutch Institute for Fundamental Energy Research - Association EURATOM-FOM, \\ Nieuwegein, The Netherlands \\ ${ }^{4}$ Max Planck Institute for Plasma Physics, Boltzmannstr. 2, 85748 Garching, Germany
}

\begin{abstract}
Extensive linear and non-linear gyrokinetic simulations and linear MHD analyses performed for JET discharges with improved confinement have shown that the large population of fast ions found in the plasma core under particular heating conditions has a strong impact on core microturbulence and edge MHD by reducing core ion heat fluxes and increasing pedestal pressure in a feedback mechanism. In the case of the ITER Like Wall (ILW), it is shown how this mechanism plays a decisive role for the transition to advanced regimes and it can explain the weak power degradation obtained in dedicated power scans. The mechanism is found to be highly dependent on plasma triangularity as it changes the balance between the improvement in the plasma core and the edge. The feedback mechanism can play a similar role in the ITER hybrid scenario as in the JET discharges analyzed due to its high triangularity plasmas and the large amount of fast ions generated in the core by the heating systems and the alpha power.
\end{abstract}

\section{Introduction}

The tokamak concept aims for the confinement of high temperature plasmas by magnetic fields. However, the high thermal energy confined is degraded by two main mechanisms: small-scale instabilities (microturbulence), and by larger-scale instabilities that can be described by MagnetoHydroDynamics (MHD). Microturbulence driven by ion temperature gradients, referred to as the Ion Temperature Gradient (ITG) mode [1], are driven linearly unstable when the logarthimic ion temperature gradients are above a critical threshold, defined by $\mathrm{R} / \mathrm{L}_{\mathrm{Ti}}>\mathrm{R} / \mathrm{L}_{\mathrm{Ti}}$,crit, where $L_{T i}=-T_{i} / \nabla T_{i}$ is the ion temperature gradient length and the tokamak major radius $\mathrm{R}$ is a normalizing factor. ITGs are typically responsible for the ion heat transport observed in confined plasmas under standard conditions, while MHD significantly restricts the access to high plasma pressure when the magnetic topology is broken at some critical pressure or pressure gradient values. Therefore, reducing the impact of these transport mechanisms is a key point in order to increase the energy confinement time in magnetically confined plasmas.

Hybrid or advanced inductive scenarios [2], characterized by high confinement, have high pressure with limited or few MHD events and therefore may extrapolate promisingly to future fusion reactor devices. The increased confinement is likely due to stability improvements both in the plasma core and at the edge, where a higher pedestal confinement is obtained compared to the standard inductive H-mode scenarios. The importance of fast ions for obtaining hybrid regimes, has been already pointed out for JET plasmas [3] where it was shown that these particles substantially contributed to the high pressure and pressure gradients typical of those regimes, and made the plasma less paramagnetic (or more diamagnetic) by significantly increasing $\beta_{p}=2 \mu_{0}\left\langle P>/\left\langle B_{\theta}^{2}\right\rangle\right.$, with $\langle\mathrm{P}\rangle$ the average pressure and $B_{\theta}$ the poloidal magnetic field, to the $\beta_{p}>1$ region, which is expected for hybrid regimes at JET [4,5]. It was also shown that there is interplay between the core and edge regions which drives the improved confinement. However, the exact physical mechanisms which allow the appearance of high pressure gradients and the interplay with fast ions and the edge were still mostly

\footnotetext{
* See the Appendix of F. Romanelli et al., Proceedings of the 25th IAEA Fusion Energy Conference 2014, Saint Petersburg, Russia
} 
unknown. Previous linear microinstability analysis has already shown that fast ions can stabilize ITG modes in the plasma core due to the fast ion dilution in thermal species [6], the modification of Shafranov-shift [7] or the electromagnetic stabilization due to a local increase of the pressure gradient [8], which is enhanced non-linearly [9]. However, none of these effects can explain by themselves the increased confinement in the edge region obtained in advanced inductive scenarios, like JET [10], and how that improvement affects the core. The dominant impact of increased edge pressure for leading to the high thermal pressure obtained in advanced inductive scenarios has been demonstrated in specific regimes [11]. However, whether an improvement in the edge stability leads to a higher core pressure or an increase in pressure is a cause for improved edge stability is something that has not been yet clarified [12].

In this paper, two C-wall hybrid JET shots, with significant thermal improved confinement and fast ion content, are analyzed to assess the previous points. For that purpose, detailed linear and non-linear gyrokinetic and linear MHD simulations are performed at the core and edge plasma regions. The results show how - unlike thermal particles - a large population of fast ions is a key ingredient for a positive feedback between plasma core and edge which can improve thermal energy confinement in both regions.

The paper is organized as follows: in section 2 the experimental discharges from the C-wall era are discussed. In section 3 the impact of fast ions in microturbulence is analyzed, and in section 4 their impact on edge MHD and the onset of a feedback with the core is discussed. In section 5 the impact of the previous findings on the low thermal energy confinement time degradation obtained in different ILW power scans is discussed. In section 6, an extrapolation to ITER is shown. Finally some conclusions are discussed in section 7.

\section{Experimental data. C-wall discharges}
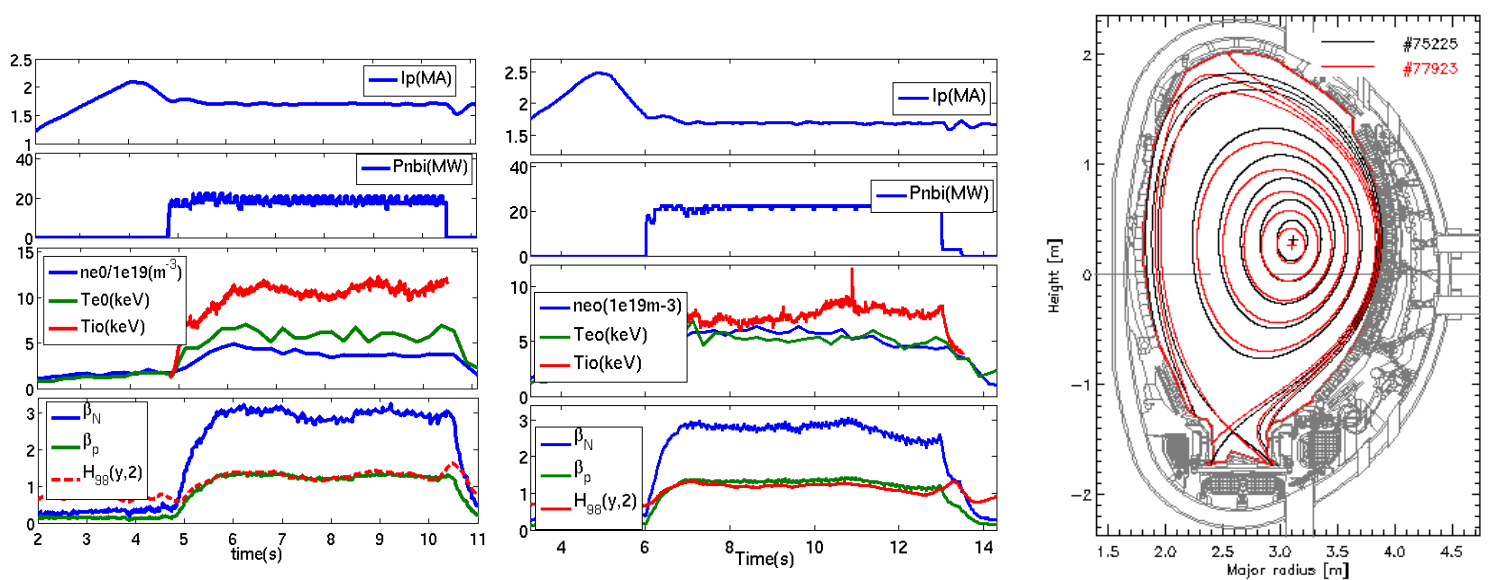

Figure 1. Time evolution for discharge \#75225 (left), for \#77923 (center), and the EFIT calculated magnetic equilibrium for both shots (right).

Two representative discharges of JET hybrid scenarios from the C-wall era, discharge \#75225 [10] at low triangularity, and \#77923 [3], at high triangularity, were analyzed. The time evolution of the main parameters is shown in figure 1 whereas a full summary is found in Tab.I. In both cases, a high input Neutral Beam Injection (NBI) power is combined with low average density and Greenwald fraction for achieving both a high beta, $\beta=2 \mu_{0}<P>/ B^{2}$, where $\mathrm{B}$ is the magnetic field, as well as a thermal energy enhancement factor relative to the IPB98(y,2) scaling [13], $\mathrm{H}_{98}(\mathrm{y}, 2)$, higher than 1. The remarkable difference between the two discharges is the upper triangularity: $\delta_{\mathrm{u}}=0.16$ for $\# 75225$ and $\delta_{\mathrm{u}}=0.38$ for $\# 77923$. This is visible from the EFIT calculated magnetic equilibrium, as shown in figure 1, and has an impact on the overall plasma behavior. Whereas the $\mathrm{H}_{98}(\mathrm{y}, 2)$ factor is similar in both cases, 
the average density and the pedestal pressure are both higher for \#77923. This feature has an impact on the core profiles as well as on the fast ions generated by the NBI system. In particular, the ion temperature has a steepened profile at $\rho=0.33$ for $\# 75225, \mathrm{R} / \mathrm{L}_{\mathrm{Ti}}=6.25$, however $\mathrm{R} / \mathrm{L}_{\mathrm{Ti}}=5.7$ for $\# 77923$. In order to assess the possible differences on the fast ion distribution for both discharges, interpretative simulations were carried out with the CRONOS [14] suite of integrated modeling codes. The fast ion content for NBI-driven fast ions was calculated by orbit following Monte Carlo codes, NEMO/SPOT [15]. The q profile used for the calculations was obtained by equilibrium reconstructions constrained by MSE angles and total pressure. In figure 2, the total, thermal, and fast ion pressures, as well as the fast ion and electron density profiles, and the magnetic equilibrium are shown for both discharges. The fast particles change the total plasma energy content by changing the core pressure profile. This change is stronger for \#75225, as the normalized beta rises from $\beta_{N, t h}^{75225}=2.13$ to $\beta_{N}^{75225}=2.9$ whereas for $\# 77923$ it is from $\beta_{N, t h}^{77923}=2.46$ to $\beta_{N}^{77923}=2.8$. The ratio of thermal and fast ion densities is also modified as $\mathrm{n}_{\text {fast }} / \mathrm{n}_{\mathrm{e}} \sim 0.13$ for $\# 75225$ and $\mathrm{n}_{\text {fast }} / \mathrm{n}_{\mathrm{e}} \sim 0.05$ for \#77923 at $\rho=0.33$, being $\rho$ the normalized toroidal magnetic flux. The difference on the fast ion population has an impact on magnetic equilibrium and in particular on the Shafranov-shift, as shown in figure 2 , which increases by $\Delta_{75225}^{\text {Shafrano-Shit }} \approx 4.0 \mathrm{~cm}$ whereas the increase is more modest for \#77923, $\Delta_{77923}^{\text {Shafranov-Shift }} \approx 2.0 \mathrm{~cm}$. Finally, the pressure gradient in the core region is also modified. A parameter of merit of the local pressure modification is $\alpha=-R q^{2} d \beta / d r$, which increases from $\alpha_{t h}^{75225}(\rho=0.33)=0.35$ up to $\alpha^{75225}(\rho=0.33)=0.52 \quad$ and $\alpha_{t h}^{75225}(\rho=0.33)=0.28$ up to $\alpha^{75225}(\rho=0.33)=0.34$. The impact of the fast ions for both discharges, and the analysis of the similitudes and differences will be analyzed in the following sections.

\begin{tabular}{c|ccccccc|} 
Shot & Ip (MA) & $\mathrm{Bt}(\mathrm{T})$ & $\mathrm{q}_{95}$ & $\kappa / \delta$ & $\beta_{\mathrm{N}} / \beta_{\mathrm{N}, \mathrm{th}}$ & $\mathrm{H}_{98}(\mathrm{y}, 2)$ & $\mathrm{P}_{\mathrm{tot}}(\mathrm{MW})$ \\
\hline 75225 & 1.7 & 2.0 & 4.0 & $1.64 / 0.23$ & $2.93 / 2.13$ & 1.30 & 17 \\
77923 & 1.7 & 2.0 & 4.0 & $1.70 / 0.38$ & $2.80 / 2.46$ & 1.30 & 21 \\
84792 & 1.4 & 1.7 & 4.4 & $1.63 / 0.27$ & $2.85 / 2.45$ & 1.20 & 13 \\
84798 & 1.4 & 1.7 & 3.9 & $1.63 / 0.25$ & $1.43 / 1.30$ & 0.93 & 6.0 \\
ITER & 12 & 5.3 & 4.30 & $1.80 / 0.40$ & $3.0 / 2.50$ & 1.30 & 73
\end{tabular}

TABLE I. Main characteristics of the discharges analyzed in this letter. Ip is the total current, $\mathrm{Bt}$ the toroidal magnetic field, $\kappa$ elongation, $\delta$ triangularity, $\beta_{\mathrm{N}=} \beta \mathrm{aB} / \mathrm{Ip}$ normalized beta (with a the plasma minor radius), $\beta_{\mathrm{N}, \mathrm{th}}$ normalized thermal beta $\mathrm{H}_{98}(\mathrm{y}, 2)$ thermal confinement factor, $\mathrm{P}_{\text {tot }}$ injected power.
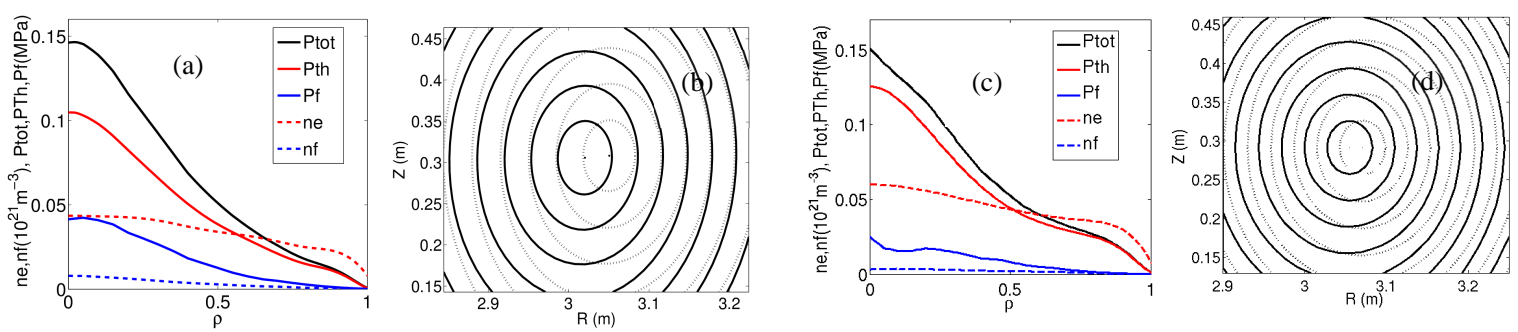

Figure 2. Total (Ptot), thermal (Pth), fast ion (Pf) pressure and electron (ne) and fast ion (nf) density profiles for the shots 75225 (a) and 77923 (c). Plasma equilibrium with fast ions (dashed) and without fast ions (solid) for the shots 75225 (b) and $77923(d)$ 


\section{Mictroturbulence analysis of $\mathbf{C}$-wall discharges}

The impact of the fast ions on core microturbuelnce is analyzed by means of the GENE gyrokinetic code [16]. GENE solves the gyrokinetic Vlasov equation, coupled selfconsistently to Maxwell's equations, within a $\delta f$ formulation. The code employs field line coordinates, where $\mathrm{x}$ is the radial coordinate, $\mathrm{z}$ is the coordinate along the field line, and $\mathrm{y}$ is the binormal coordinate thus adapting in an optimum way to the highly anisotropic plasma microturbulence. All simulations were carried out in flux tubes, i.e., in very small radial domain where the local limit is considered. The geometry used was calculated by the HELENA code [17] based on the interpretative analysis of the discharges. Collisions are modeled using a linearized Landau-Boltzmann operator. Both linear and nonlinear initial value simulations were performed. Typical grid parameters were as follows: perpendicular box sizes $[\mathrm{Lx}, \mathrm{Ly}]=[250,125]$ in units of the ion Larmor radii, perpendicular grid discretization [nx, nky] $=[256,32], 32$ point discretization in the parallel direction, 48 points in the parallel velocity direction, and 12 magnetic moments. The comparatively high Lx and $\mathrm{nx}$ values were necessary to satisfy the boundary conditions in the low magnetic shear simulations at low radii. Perpendicular and parallel magnetic fluctuations were included in the simulations. This cannot be neglected, due to the relatively high $\beta$, and is important for setting the strength of electromagnetic coupling, as seen in dedicated checks.

The turbulent linear growth rates $\gamma$ and flow shear rate, $\gamma_{\mathrm{E}}$, are in units of $\mathrm{c}_{\mathrm{s}} / \mathrm{R}$, with $c_{s}=\sqrt{T_{e} / m_{i}}$ and $\mathrm{m}_{\mathrm{i}}$ the main ion mass. The fast particle distribution function was approximated as Maxwellian, taking the average energy of the fast ion slowing-down distribution to derive the temperature to be $\mathrm{T}_{\text {fast }}=35 \mathrm{keV}$ for $\# 75225$ and $\mathrm{T}_{\text {fast }}=27 \mathrm{keV}$ for \#77923. For the gyrokinetic calculations excluding the energetic ions, the equilibrium was recalculated with only the thermal pressure, and then used for the simulations. The dimensionless parameters of the discharge at $\rho=0.33$ fed into the gyrokinetic calculations are summarized in Tab. II. The selected time for \#75225 is $\mathrm{t}=6.03 \mathrm{~s}$ and $\mathrm{t}=7 \mathrm{~s}$ for \#77923.

\begin{tabular}{c|ccccccccc} 
Shot & $\mathrm{s}$ & $\mathrm{q}$ & $\mathrm{T}_{\mathrm{e}} / \mathrm{T}_{\mathrm{i}}$ & $\mathrm{R} / \mathrm{L}_{\mathrm{Ti}}$ & $\mathrm{R} / \mathrm{L}_{\mathrm{Te}}$ & $\mathrm{R} / \mathrm{L}_{\mathrm{Ne}}$ & $\mathrm{T}_{\text {fast }} / \mathrm{T}_{\mathrm{e}}$ & $\mathrm{R} / \mathrm{L}_{\text {Tfast }}$ & $\mathrm{R} / \mathrm{L}_{\mathrm{Nfast}}$ \\
\hline 75225 & 0.15 & 1.16 & 0.67 & 6.2 & 4.3 & 2.6 & 7.2 & 0.4 & 7.1 \\
77923 & 0.22 & 1.09 & 0.87 & 5.7 & 4.6 & 1.1 & 6.7 & 3.0 & 4.0 \\
84792 & 0.13 & 1.13 & 0.85 & 4.2 & 3.9 & 1.3 & 6.4 & 3.8 & 4.6 \\
84798 & 0.14 & 1.11 & 1.17 & 3.6 & 4.3 & 0.6 & 7.9 & 4.7 & 6.2 \\
\cline { 2 - 9 }
\end{tabular}

TABLE II. Discharge dimensionless parameters at $\rho=0.33$ used as input in simulations
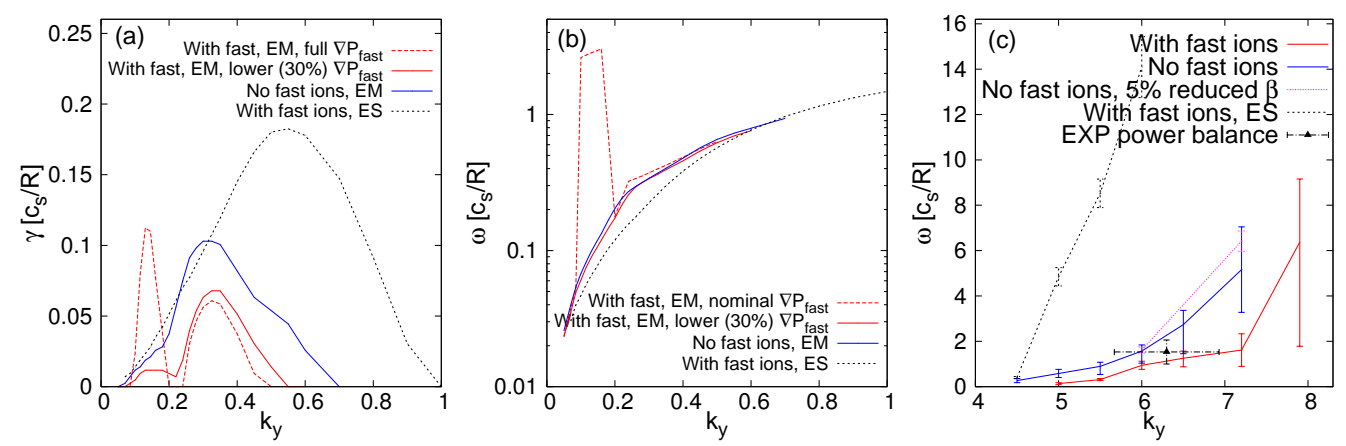

Figure 3 Linear growth rates and frequencies for discharge 75225 (a,b) Ion heat flux from nonlinear gyrokinetic simulations (c). The analysis was carried out at $\rho=0.33$. 
In Fig. 3, the linear growth rates and frequencies are shown for the nominal experimental value of $\mathrm{R} / \mathrm{L}_{\mathrm{Ti}}=6.25$ for various assumptions in the input parameters for the discharge $\# 75225$. In order to identify the nature of the underlying modes, the ExB flow shear rate was set to zero. Over a wide normalized wavenumber range, $k_{y}=k_{y} \rho_{s}$ where $\rho_{s}$ is the ion gyroradius with respect to the sound speed, ITG modes are unstable. The maximum growth rate is reduced by $\approx 30 \%$ when including fast ions in the system. When including the nominal fast ion pressure gradient, an additional mode is apparent at $k_{y}=0.1-0.15$. This mode is stabilized when reducing the fast ion pressure gradient by $30 \%$ and it has a significantly higher frequency than the ITG modes, at $\omega \approx 2.5$. This is within $5 \%$ of the corresponding GAM frequency as determined by a Rosenbluth-Hinton test [18]. This mode is identified as a $\beta$-induced Alfvén Eigenmode (BAE) [19], which is known to be degenerate with the GAM frequency and destabilized by fast ions. This mode share characteristics and has similar frequency as the Kinetic Ballooning Modes (KBM), and we will refer to it as a generalized BAE/KBM electromagnetic mode. Therefore, at this radial position, the turbulence regime is found to be in the direct vicinity of the ITG-BAE/KBM boundary. A nonlinear $\mathrm{R} / \mathrm{L}_{\mathrm{Ti}}$ scan with and without fast ions, is shown in Fig. 3. The reduced fast ion pressure gradient is maintained throughout this scan. This is consistent with an assumption that the fast ion pressure is clamped by the manifestation of the BAE-like modes which are known to drive fast ion transport. This reduced fast ion pressure gradient can be obtained from the SPOT modeling when assuming a fast ion diffusion coefficient of $D_{\text {fast }}=0.5 \mathrm{~m}^{2} / \mathrm{s}$.

The experimental toroidal rotation was taken into account by including the ExB flow shear rate, $\gamma_{E}=0.2$, and the according parallel flow shear. The heat flux obtained is normalized by the gyroBohm normalization factor, $q_{G B}=T_{i}^{2.5} n_{i} m_{i}^{0.5} / e^{2} B^{2} R^{2}$, with $\mathrm{n}_{\mathrm{i}}$ is the ion density. Here, the fast ions provide an improvement of $\approx 10-25 \%$ on $\mathrm{R} / \mathrm{L}_{\mathrm{Ti}}$ for the same heat flux. This improvement is obtained in combination of electromagnetic effects as otherwise, if the fast ions are included but electrostatic simulation are performed, the heat fluxes are much higher than the one obtained by power balance. This shows the importance of performing predicting simulations including both electromagnetic effects and all the active species in the plasma.

When including fast ions, the ion heat flux rapidly increases for $\mathrm{R} / \mathrm{L}_{\mathrm{Ti}}>7.2$ and the fast ions are no longer stabilizing. This is correlated with a significant increase in the fast ion transport. Linear analysis shows that the BAE/KBM modes are destabilized in this high $\mathrm{R} / \mathrm{L}_{\mathrm{Ti}}>7.2$ regime, even with the reduced fast pressure gradient. These modes have a strong resonant interaction with both thermal and energetic ions. The consistency of the simulation output and power balance values, in a BAE/KBM marginal regime, suggests that a self- limitation mechanism may be active, whereby the fast ion pressure gradient is maintained at a level consistent with BAE marginality.
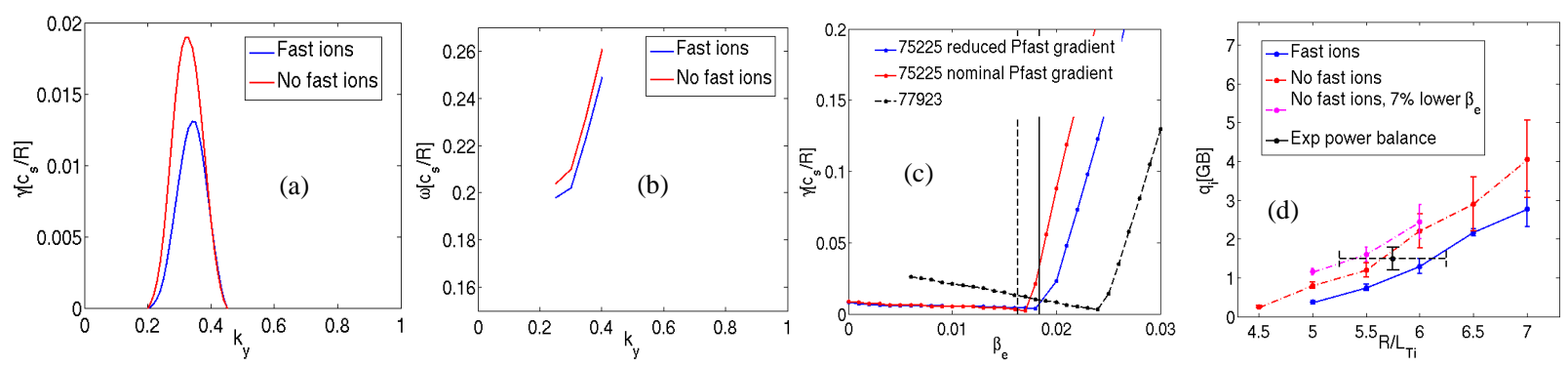

Figure 4 Linear growth rates and frequencies for discharge $77923(\mathrm{a}, \mathrm{b})$ Linear growth rate dependence on $\beta_{\mathrm{e}}$. Vertical lines represent experimental data for 77923 (dashed) and 75225 (solid) (c). Ion heat flux from nonlinear gyrokinetic simulations (d). The analysis was carried out at $\rho=0.33$.

The impact of the fast ions has been as well analyzed for the discharge 77923. In Fig. 4, the linear growth rates and frequencies are shown for the nominal experimental value of 
$\mathrm{R} / \mathrm{L}_{\mathrm{Ti}}=5.7$. Unlike the previous case, no $\mathrm{BAE} / \mathrm{KBM}$ modes are obtained and only ITG modes are unstable in a narrow $k_{y}=0.2-0.45$ region. This is consistent with the fact the pressure gradient is lower and the magnetic shear higher for this discharge at the selected point. In order to demonstrate that the $\mathrm{BAE} / \mathrm{KBM}$ limit is indeed different for both discharges a scan on the electron beta, $\beta_{\mathrm{e}}$, has been performed and shown in figure 4 . In spite of the fact that both discharges have similar $\beta$, the discharge \#75225 lies at the boundary between ITG and $\mathrm{BAE} / \mathrm{KBM}$ whereas the $\# 77923$ is at $60 \%$.

The maximum growth rate is reduced by $30 \%$ when the fast ions are included in this case, a value similar to the one obtained for the low triangularity discharge. A nonlinear $\mathrm{R} / \mathrm{L}_{\mathrm{Ti}}$ scan was performed as well with and without fast ions, as shown in Fig. 4. The experimental toroidal rotation was taken into account by including the $E x B$ flow shear rate, $\gamma_{E}=0.15$, and the according parallel flow shear. Here, the fast ions provide an improvement of $10 \%$ of the heat flux, in the range of $\mathrm{R} / \mathrm{L}_{\mathrm{Ti}}$ analyzed. The agreement between the heat flux obtained and the one calculated from power balance is still quite good. For this discharge, the improved heat flux at the experimental $\mathrm{R} / \mathrm{L}_{\mathrm{Ti}}$ is lower than for the discharge $\# 75225$, which reached $25 \%$. One possible reason is the proximity to the KBM limit. As shown in figure 4 and in [20], the ITG stabilization by electromagnetic effects is stronger when the plasma is closer to the $\mathrm{KBM} / \mathrm{BAE}$ limit. However, electromagnetic effects, in particular the ones obtained in nonlinear simulations are still significant for this discharge. In figure 5, ion heat fluxes and linear growth rates are compared for different $\beta_{\mathrm{e}}$. Whereas the linear stabilization is significant, the nonlinear one is stronger and highly contributes to the low heat fluxes obtained at high $\beta$.

Additionally, two extra simulations for both discharges, one with no ExB shear and another one with three times the nominal ExB shear, were performed. Results, presented in figure 5 show the electromagnetic effects are essential to obtain the correct ion heat fluxes for both discharges as qi significantly increases in the electrostatic case. Regarding the ExB flow shear, its effect tends to be negligible or even destabilizing when the full electromagnetic simulations are performed, however it is stabilizing for the electrostatic simulation. This confirms that the electromagnetic effects together with the fast ions have a significant stabilization effect in the plasma core, much more than ExB shear flow.
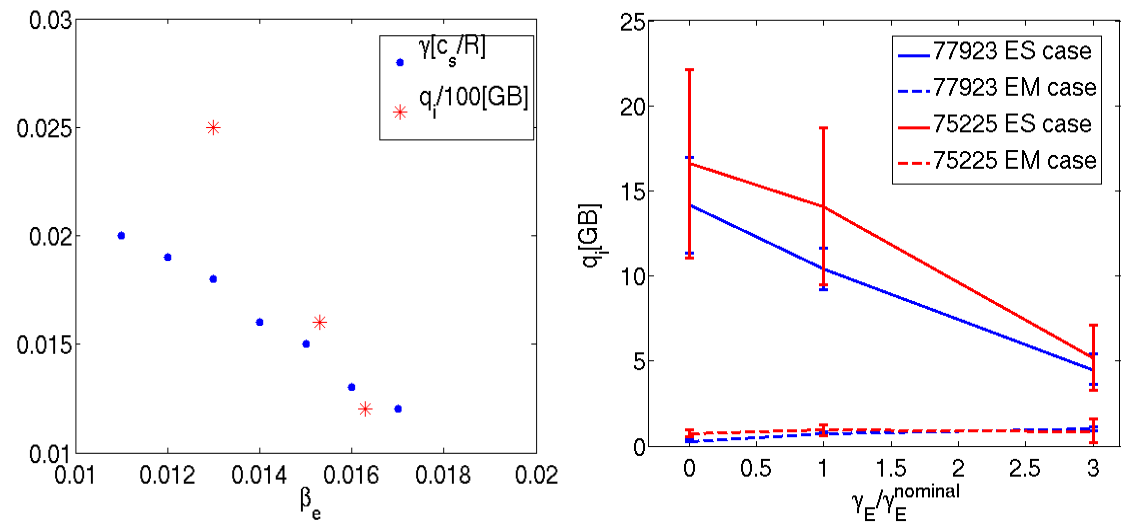

Figure 5 Growth rate and ion heat flux dependence on $\beta_{\mathrm{e}}$ for the discharge \#77923 (left) Ion heat flux dependence on $\gamma_{\mathrm{E}}$ in the case of electrostatic case (ES) and electromagnetic case (EM) (right) 


\section{Edge MHD analysis and core-edge interplay}

We now analyze the edge stability. The peeling-ballooning diagram was calculated following the procedure detailed in Ref [21]. The MHD code MISHKA [22] was used for computing linear ideal MHD stability at the edge. For this purpose, as previously, the equilibrium was calculated with and without fast ions. In the peeling-ballooning diagram, shown in figure 6 for both discharges, the experimental pedestal pressure is well predicted by the calculations and the experimental value lies on the ballooning side. This good agreement is obtained when the fast ion pressure is included, as it extends the stable region for both discharges. This shows the favorable impact of $\beta$ on the edge stability through the Shafranov-shift, as obtained from the analysis of DIII-D and JT-60U [11,23]. However, it is the fast component of $\beta$ which is mainly responsible for the improved confinement, as will be shown in next section. It is worth pointing out that, due to the plasma stiffness, even a modest improvement of the pedestal can lead to a higher overall improved thermal confinement compared with solely improving the core microturbulence as the volume affected is much higher.

Regarding the edge stabilization by fat ions, there is a fundamental difference between both discharges. While the core fast ion pressure for the discharge \#75225, which is $30 \%$ of the total energy, increases the stable region by $10 \%$, a $15 \%$ increase is found for the discharge \#77923 where just $10 \%$ of the total energy can be attributed to the fast ions. This shows the importance of the high triangularity configuration for taking advantage of the stabilization of the pedestal by increasing $\beta$.

A rough analysis of the interplay between core and edge has been studied by performing nonlinear simulations for both discharges at 5\% lower $\beta_{e}$ for \#75225 and 7\% for \#77923, assuming that a reduction of pedestal pressure obtained by removing the fast ion content leads to a less electron pressure in the core through stiffness. As shown in figure 3 and 4 , the ion heat flux increase for both discharges up to $10 \%$ due to lower electromagnetic stabilization of ITG modes. This shows that small changes in the pedestal region can be propagated and amplified in the core by electromagnetic effects. However, it should be pointed out that $\beta$ should be high enough, otherwise the electromagnetic effects are too weak and the core-edge interplay is not an effective mechanism for turbulence reduction. This point will be further clarified in section 5 .

In order to show how the fast ions are a key ingredient for both a microturbulence improvement in the core and a pedestal pressure at the edge, an alternative gyrokinetic calculation was carried out. The fast ion content was removed for the discharge \#75225 and the thermal electron and ion content and gradients were increased such that $\beta_{\mathrm{N}}$ and $\alpha$ are maintained, whereas the plasma geometry is also kept constant. In these two cases the pedestal would behave exactly the same as the Shafranov-shift is identical. The new growth rates, shown in figure 6, increase throughout the spectrum, and its maximum is 2.5 times higher compared to the case with fast ions which would result in a much higher heat flux. The key point is that the fast ions, unlike thermal particles, increase pressure in the plasma core, while simultaneously not contributing to the ITG drive and even stabilizing it (which can lead to higher thermal pressure as the ion temperature also increases). Therefore under the same conditions, in particular the same input power, a particular level of Shafranov-shift (or of pedestal improvement) is achieved much more efficiently in the presence of a high fast ion population than with pure thermal particles. Increased thermal content also increases core microturbulence limiting the global $\beta$ obtained and therefore also limiting the possible pedestal pressure attained. Finally, for these shots the relative weight of the effects varies. In the core the fast ions and electromagnetic effects have a stronger impact for \#75255 due to the lower density and vicinity to the KBM/BAE boundary. However the impact of the suprathermal pressure for edge enhancement is lower due to the low triangularity. On the 
other hand, for \#77923, the trend is the opposite. The global effect could be eventually the same for both discharges, as it happens for the ILW dedicated power scans [24], however, self-consistent simulations are required in order to confirm this point.
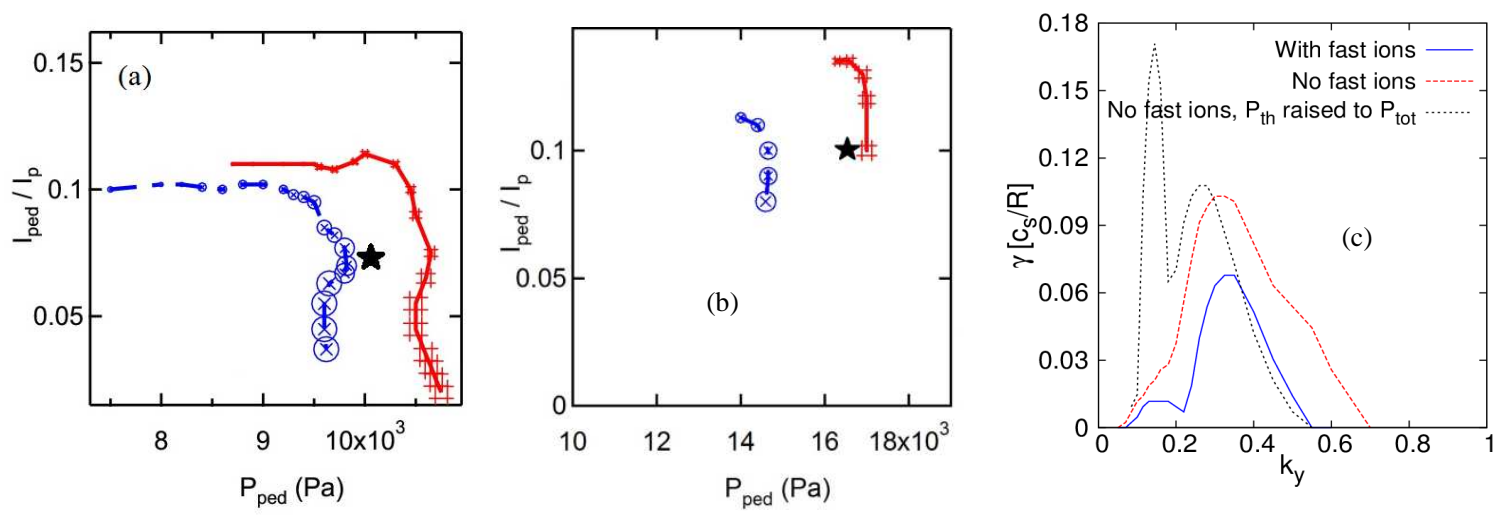

Figure 6. Peeling-ballooning boundaries for discharges 75225 (a) and 77923 (b). The experimental value is marked with a star. Large or small symbols indicate high or low toroidal mode number respectively. Linear growth rates with and without fast ions and with artificially increased thermal pressure (c).

\section{Role of fast ions and finite-beta on power degradation}

Recent power scans performed in the ILW, both at high and low triangularity, have shown that the power degradation of thermal energy confinement is weaker than the one expected for the IPB98 $(y, 2)$ scaling [24]. In these scans all the engineering parameters were kept fixed and just the power was increased. The dependence of the factor $\mathrm{H}_{98}(\mathrm{y}, 2)$ on the injected power is shown in Fig. 7. At high power, $\mathrm{H}_{98}(\mathrm{y}, 2)>1$ is obtained, which means that the thermal energy confinement is degraded less than expected from the IPB98(y,2) scaling. This can be easily explained by analyzing the energy confinement time, $\tau_{h}$, dependence on the power, $\mathrm{P}$. Whereas for the IPB98 $(\mathrm{y}, 2)$ scaling such confinement dependence is, $\tau_{h} \propto P^{-0.69}$, a much weaker dependence is obtained in the scans for both high triangularity, $\tau_{h} \propto P^{-0.25}$ and low triangularity $\tau_{h} \propto P^{-0.30}$ [24]. The deviation from the scaling is particularly evident at high power where the stored energy is much higher than the one predicted. The increasing of the pedestal pressure with power (or beta) has been shown to play an important role on this low power degradation, especially for the high triangularity cases [24], in agreement with the result obtained in section 4. However, for the low triangularity cases, with lower average and central density, core effects could play a stronger role as previously shown. Therefore two discharges from the power scan at low triangularity are selected in order to analyze the impact of fast ions on the low power degradation obtained. The main characteristics of both discharges are shown in table I and II whereas the time evolution of the injected power, $\beta_{\mathrm{N}}$ and $\mathrm{H}_{98}(\mathrm{y}, 2)$ are shown in figure7. The time window analyzed is $\mathrm{t}=45.2 \mathrm{~s}-45.5 \mathrm{~s}$ and the radial location is $\rho=0.33$.

As it happens in the $\mathrm{C}$-wall discharges previously analyzed, over a wide normalized wavenumber range, ITG modes are unstable for both discharges, as shown in figure 8 . However, for the low power shot 84798, Trapped Electron Modes (TEM) appear at $\mathrm{k}_{\mathrm{y}}>0.7$. The maximum growth rate is reduced by including the contribution of the fast ions to the pressure gradient, however, this reduction is almost negligible for the low power case whereas it is strong for the high power one, in agreement with the fact that the fast ion content increases from $10 \%$ to $20 \%$. The impact of electromagnetic effects follows the same trend. 
This suggests that these effects play an important role for the lower power degradation as the power increases.

This hypothesis is confirmed by performing nonlinear simulations as shown in figure 8 . The experimental toroidal rotation was taken into account by including the $\operatorname{ExB}$ flow shear rate and the according parallel flow shear. For the high power shot, the fast ions provide a heat flux reduction of three times for the same $\mathrm{R} / \mathrm{L}_{\mathrm{Ti}}$, whereas their impact is negligible for the low power case. The impact of electromagnetic effects follow a similar trend, however, unlike the fast ions effects, its impact is not negligible at low power. In the case of the discharge 84798, the TEM weakly contribute to the total ion heat flux.

In conclusion, the electromagnetic effects, which are enhanced by the presence of fast ion, increase with the increasing power and they can be partially responsible for the low power degradation obtained in the power scan. This, together with the increased pressure at the top of the pedestal obtained by the increased power can explain the increase of $\mathrm{H}_{98}(\mathrm{y}, 2)$ with power and the reduction of the degradation of the thermal energy confinement time at high power. Therefore, the transition to advanced scenarios at JET in both C-wall and in ILW shares the same characteristics.
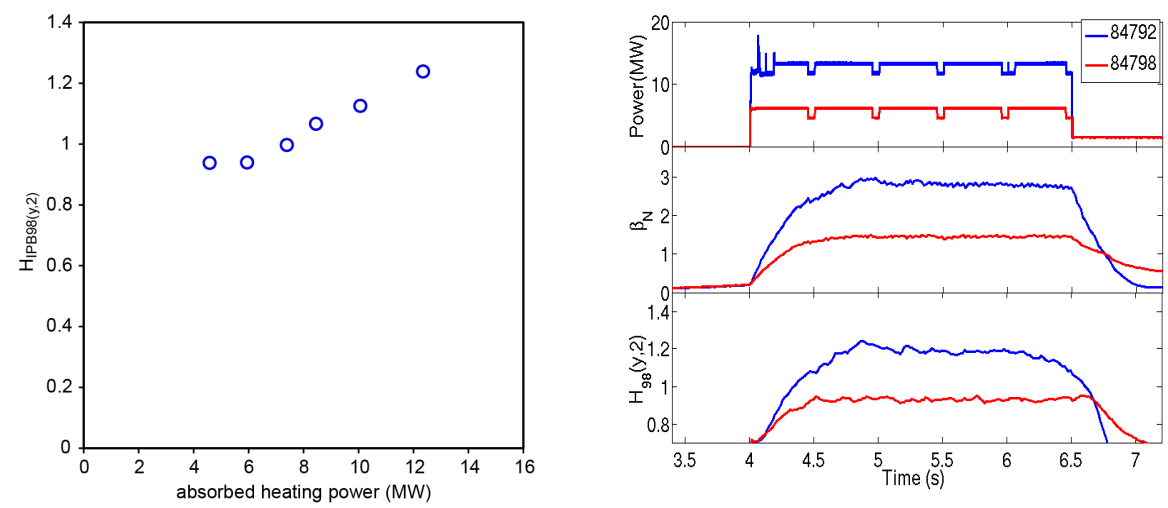

Figure 7. Dependence of the $\mathrm{H}_{98}(\mathrm{y}, 2)$ factor on the absorbed power for the low $\delta$ power scan. (Left). Time evolution for the discharges 84792 and 84798 (Right).
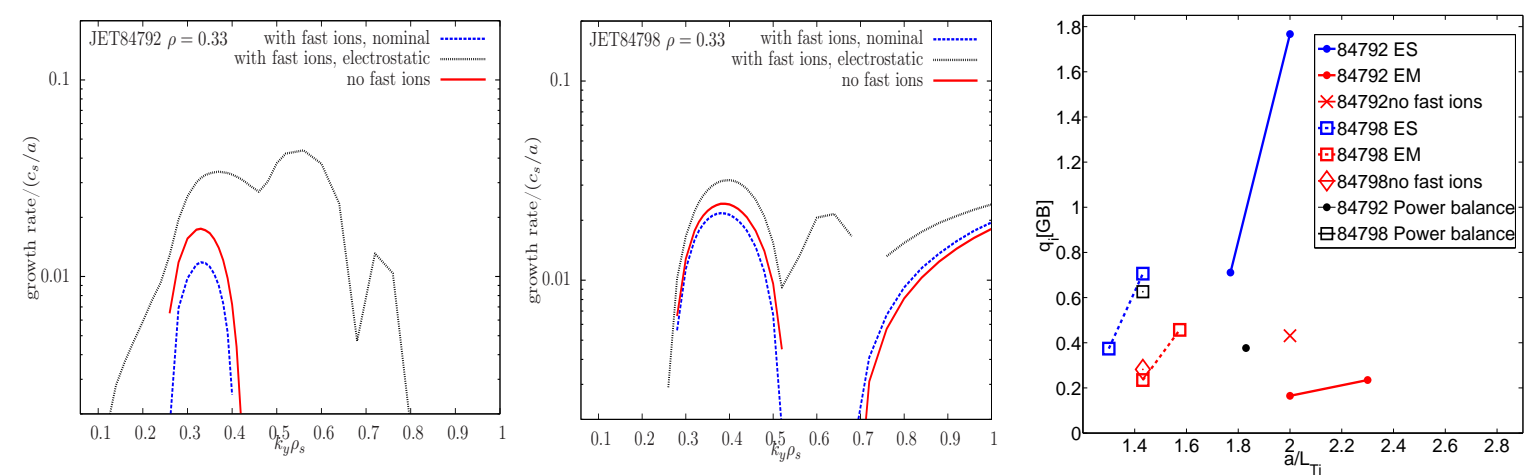

Figure 8. Linear growth rates and frequencies for discharge 84792 (Left) Linear growth rates and frequencies for discharge 847928 (center). Ion heat flux from nonlinear gyrokinetic simulations for the discharges 84792 and 84798 (right).

\section{ITER analysis}

With the aim of analyzing if the same effect can play a role in tokamaks with low external torque, as ITER, the same procedure has been applied to a typical ITER hybrid scenario [25] with the characteristics shown in table 1 . The suprathermal pressure from all the power 
sources is shown in figure 9. Although the fast ion densities associated with the fusion $\alpha-$ particles and with the NBI beams are low, $\mathrm{n}_{\text {fast, } \alpha} / \mathrm{n}_{\mathrm{e}} \sim 0.009$ and $\mathrm{n}_{\text {fast,beams }} / \mathrm{n}_{\mathrm{e}} \sim 0.006$, their pressure (and pressure gradient), is not negligible as their energy is quite high, $\mathrm{T}_{\text {fast,alpha }}=1.1$

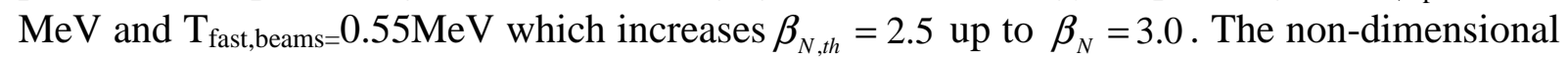
parameters used in the GENE simulations are shown in table III. Microturbulence, calculated at $\rho=0.33$, is found to be ITG and also modified by these fast ions with a growth rate reduction of up to $30 \%$. The increased $\beta$ also expands the stable peeling-ballooning region at the edge, similarly as obtained for JET (up to $10 \%$ ). This represents a significant increase of fusion power, which in turn would also increase $\beta$ in a positive feedback.

The fact that the mechanism discussed in this paper might have the same impact in ITER than in JET comes from the fact that ITER gets a maximum benefit from the core fast ions, due to the high population of $\alpha$-particles and from the stabilization of the pedestal, because of the high triangularity. Therefore, the ITER hybrid behaves as a mixture of JET low and high triangularity plasmas. This implies that, unlike rotational flow shear, the role played by fast ions in ITER and future tokamak reactors can have the same impact as in present day experiments, primarily due to the $\alpha$-particles, which will be the main heating mechanism. However, nonlinear simulations are required in order fully address the impact of electromagnetic and fast ions effects in ITER. This will be carried out in the future.

\begin{tabular}{c|cccccccccccc} 
Shot & $\mathrm{s}$ & $\mathrm{q}$ & $\mathrm{T}_{\mathrm{e}} / \mathrm{T}_{\mathrm{i}}$ & $\mathrm{R} / \mathrm{L}_{\mathrm{Ti}}$ & $\mathrm{R} / \mathrm{L}_{\mathrm{Te}}$ & $\mathrm{R} / \mathrm{L}_{\mathrm{Ne}}$ & $\mathrm{T}_{\mathrm{f}, \text { beams }} / \mathrm{T}_{\mathrm{e}}$ & $\mathrm{R} / \mathrm{L}_{\mathrm{Tf}, \text { beams }}$ & $\mathrm{R} / \mathrm{L}_{\mathrm{Nf}, \text { beams }}$ & $\mathrm{T}_{\mathrm{f}, \alpha} / \mathrm{T}_{\mathrm{e}}$ & $\mathrm{R} / \mathrm{L}_{\mathrm{Tf}, \alpha}$ & $\mathrm{R} / \mathrm{L}_{\mathrm{Nf}, \alpha}$ \\
\hline ITER & 0.24 & 1.17 & 1.09 & 3.4 & 2.9 & 1.9 & 22.5 & 1.85 & 13.1 & 41.3 & 0.94 & 9.23
\end{tabular}

TABLE III. Discharge dimensionless parameters at $\rho=0.33$ used as input in simulations
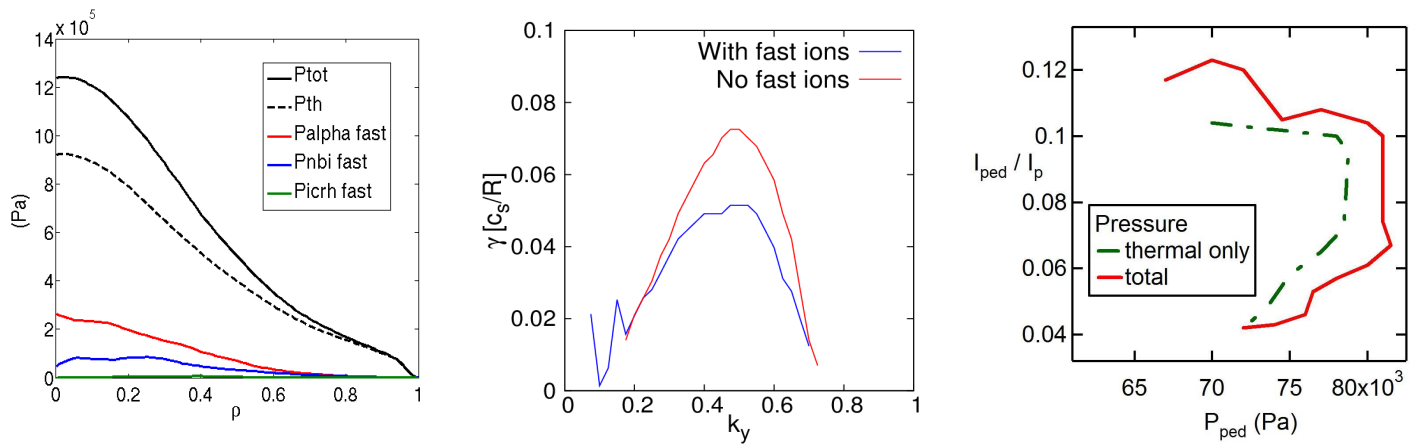

Figure 9. Total (Ptot), Thermal (Pth), alpha fast ion (Palpha fast), nbi fast ion (Pnbi fast) and Ion Cyclotron Resonant Heating (ICRH) fast ion (Picrh fast) pressure profiles (left). Linear growth rates with and without fast ions for the ITER hybrid scenario at $\rho=0.33$ (center). Peeling-ballooning boundaries with and without fast ions for ITER (right).

\section{Conclusions}

We have presented evidence of the simultaneous modification by fast ions of microturbulence and MHD by performing gyrokinetic and MHD analyses to two JET hybrid shots at high and low triangularity. In the core, mictroturbulence is reduced by fast ions when electromagnetic effects are taken into account whereas the ExB flow shear has been shown to play a minor effect. At the same time, the fast ions increase the total core pressure without increasing the turbulence drive. This leads to an improved edge pedestal pressure by means of the increased Shafranov-shift in a manner unachievable by pure thermal pressure, which is strongly limited by microturbulence. A positive core-edge feedback is therefore established and it is expected to be efficient until $\mathrm{BAE} / \mathrm{KBM}$ modes are destabilized due to the strong plasma pressure 
gradient obtained, which would increase both thermal and fast ion transport. However, it has been shown that this interplay is highly dependent on the magnetic geometry as the relative improvement in the core and at the edge, and their interplay, depends on triangularity and plasma density.

This feed-back mechanism has extraordinary consequences for the well-known degradation of the thermal energy confinement time with power. The fact that the fraction of fast ions and core $\beta$ increase with power make the confinement to decrease slower than the IPB98 $(y, 2)$ scaling. This feature has an important impact on 0-D analyses of tokamak devices for which $\beta$ is important for generating enough fusion power, such as DEMO or the fusion tokamak reactor, for which present day studies might be too pessimistic.

In fact, in ITER, this effect can play a similar role as in present day experiments, owing to the high level of fast ions born from fusion reactions, which highly increase the pressure gradient in the plasma core, and therefore can compensate for the low rotation expected due to the high density required for producing sufficient fusion energy.

Acknowledgements. This work was supported by EURATOM and carried out within the framework of the European Fusion Development Agreement. The views and opinions expressed herein do not necessarily reflect those of the European Commission. This work has been carried out within the framework of the EUROfusion Consortium and has received funding from the European Union's Horizon 2020 research and innovation programme under grant agreement number 633053. The simulations presented in this work were carried out using the HELIOS supercomputer system at Computational Simulation Centre of International Fusion Energy Research Centre (IFERC-CSC), Aomori, Japan.

\section{References}

[1] F. Romanelli, Phys. Fluids B 1, 1018 (1989).

[2] T.C. Luce et al., Nucl. Fusion 43, 321 (2003).

[3] J. Garcia and G. Giruzzi Nucl. Fusion 53, 043023 (2013).

[4] M.N.A. Beurskens et al 2013 Nucl. Fusion 53013001

[5] J. Garcia and G. Giruzzi, Phys. Rev. Lett. 104, 205003 (2010).

[6] G. Tardini et al. Nucl. Fusion 47, 280 (2007).

[7] C. Bourdelle, et al., Nucl. Fusion 45, 110 (2005).

[8] M. Romanelli et al., Plasma Phys. Control. Fusion 52, 045007 (2010).

[9] J. Citrin et al., Phys. Rev. Lett. 111, 155001 (2013).

[10] J. Hobirk et al., Plasma Phys. Control. Fusion 54095001 (2012).

[11] Y. Kamada et al., Plasma Phys. Control. Fusion 44 A279-A286 (2002).

[12] C.F. Maggi et al., Nucl. Fusion 47, 535 (2007).

[13] ITER Physics Basis, Nucl. Fusion 39, 2175 (1999).

[14] J. F. Artaud et al., Nucl. Fusion 50, 043001 (2010).

[15] M. Schneider et al., Nucl. Fusion 51, 063019 (2011).

[16] F. Jenko, W. Dorland, M. Kotschenreuther and B.N. Rogers, Phys. Plasmas 7, 1904 (2000).

[17] G.T.A. Huysmans et al., CP90 Conference on Comp.Physics, Word Scientific Publ. Co. 1991, p.371.

[18] M. N. Rosenbluth and F.L. Hinton, Phys. Rev. Lett. 80,724 (1998).

[19] F. Zonca et al., Plasma Phys. Control. Fusion 38, 2011 (1996).

[20] J. Citrin et al. accepted in Plasma Phys. Control. Fusion.

[21] P. Maget et al., Nucl. Fusion 53093011 (2013).

[22] G.T.A. Huysmans et al., Plasma Phys. Control. Fusion 84292 (2001). 
[23] P. Snyder et al., Nucl. Fusion 47, 961 (2007).

[24] C. Challis et al., IAEA FEC 13-18 October, St. Petersburg EX/9-3

[25] K. Bassiguir et al., Plasma Phys. Control. Fusion 55, 125012 (2013). 